\title{
HYPOCOERCIVITY AND SUB-EXPONENTIAL LOCAL EQUILIBRIA
}

\author{
E. BOUIN, J. DOLBEAULT, L. LAFLECHE, C. SCHMEISER
}

\begin{abstract}
Hypocoercivity methods are applied to linear kinetic equations without any space confinement, when local equilibria have a sub-exponential decay. By Nash type estimates, global rates of decay are obtained, which reflect the behavior of the heat equation obtained in the diffusion limit. The method applies to Fokker-Planck and scattering collision operators. The main tools are a weighted Poincaré inequality (in the Fokker-Planck case) and norms with various weights. The advantage of weighted Poincaré inequalities compared to the more classical weak Poincaré inequalities is that the description of the convergence rates to the local equilibrium does not require extra regularity assumptions to cover the transition from super-exponential and exponential local equilibria to sub-exponential local equilibria.
\end{abstract}

\section{INTRODUCTION}

This paper is devoted to a hypocoercivity method designed for obtaining decay rates in weighted $\mathrm{L}^{2}$ norms of the solution to the Cauchy problem

$$
\left\{\begin{array}{l}
\partial_{t} f+v \cdot \nabla_{x} f=\mathrm{L} f, \\
f(0, x, v)=f^{\text {in }}(x, v),
\end{array}\right.
$$

for a distribution function $f(t, x, v)$, with position $x \in \mathbb{R}^{d}$, velocity $v \in \mathbb{R}^{d}$, and time $t \geq 0$. The linear collision operator $\mathrm{L}$ acts only on the velocity variable and its null space is assumed to be one-dimensional and spanned by the local equilibrium $F$, a probability density of the form

$$
F(v)=C_{\alpha} e^{-\langle v\rangle^{\alpha}}, \quad v \in \mathbb{R}^{d}, \quad \text { with } C_{\alpha}^{-1}=\int_{\mathbb{R}^{d}} e^{-\langle v\rangle^{\alpha}} \mathrm{d} v,
$$

where we use the notation

$$
\langle v\rangle:=\sqrt{1+|v|^{2}}
$$

Our results will be concerned with the sub-exponential case $0<\alpha<1$, as opposed to the exponential $(\alpha=1)$ and super-exponential $(\alpha>1$, including the Gaussian with $\alpha=2$ ) cases. This specific choice of the form of the equilibrium is for notational convenience in the proofs. The results can easily be extended to more general

Date: December 10, 2019.

2010 Mathematics Subject Classification. Primary: 82C40. Secondary: 76P05; 35H10; 35Q84.

Key words and phrases. Hypocoercivity; linear kinetic equations; Fokker-Planck operator; scattering operator; transport operator; weighted Poincaré inequality; weak Poincaré inequality; subexponential local equilibria; micro/macro decomposition; diffusion limit; decay rate. 
distributions $F$, satisfying

$$
\alpha:=\lim _{|v| \rightarrow+\infty} \frac{\log (-\log F(v))}{\log |v|} \in(0,1) .
$$

We shall consider two types of collision operators, either the Fokker-Planck operator

$$
\mathrm{L}_{1} f=\nabla_{v} \cdot\left(F \nabla_{v}\left(F^{-1} f\right)\right),
$$

or the scattering operator

$$
\mathrm{L}_{2} f=\int_{\mathbb{R}^{d}} \mathrm{~b}\left(\cdot, v^{\prime}\right)\left(f\left(v^{\prime}\right) F(\cdot)-f(\cdot) F\left(v^{\prime}\right)\right) \mathrm{d} v^{\prime} .
$$

We assume local mass conservation

$$
\int_{\mathbb{R}^{d}} \mathrm{~L} f \mathrm{~d} v=0
$$

which always holds for $L=L_{1}$, and also for $L=L_{2}$ under the assumption

$$
\int_{\mathbb{R}^{d}}\left(\mathrm{~b}\left(v, v^{\prime}\right)-\mathrm{b}\left(v^{\prime}, v\right)\right) F\left(v^{\prime}\right) \mathrm{d} v^{\prime}=0 .
$$

Note that micro-reversibility, i.e., the symmetry of $\mathrm{b}$, is not required.

Further assumptions on the cross-section b that will be given below guarantee that the operators $L_{1}$ and $L_{2}$ are responsible for the same type of asymptotic behavior. As a motivation, the relaxation properties of $L_{1}$ can be made transparent by the symmetrizing transformation $f=g \sqrt{F}$, leading to the transformed operator

$$
g \mapsto \frac{1}{\sqrt{F}} \nabla_{v} \cdot\left(F \nabla_{v} \frac{g}{\sqrt{F}}\right)=\Delta_{v} g-\nu_{1}(v) g
$$

with the collision frequency

$$
\nu_{1}(v)=\frac{\Delta_{v} F}{2 F}-\frac{\left|\nabla_{v} F\right|^{2}}{4 F^{2}} \approx \frac{\alpha^{2}}{4}|v|^{-2(1-\alpha)} \quad \text { as }|v| \rightarrow \infty .
$$

Partially motivated by this, we assume the existence of constants $\beta, \overline{\mathrm{b}}, \underline{\mathrm{b}}>0, \gamma \geq 0$, with $\gamma \leq \beta, \gamma<d$, such that

$$
\underline{\mathrm{b}}\langle v\rangle^{-\beta}\left\langle v^{\prime}\right\rangle^{-\beta} \leq \mathrm{b}\left(v, v^{\prime}\right) \leq \overline{\mathrm{b}} \min \left\{\left|v-v^{\prime}\right|^{-\beta},\left|v-v^{\prime}\right|^{-\gamma}\right\} .
$$

The upper bound with the restriction on the exponent $\gamma$ serves as a local integrability assumption. Hypotheses (H1) and (H2) permit the choice $\mathrm{b}\left(v, v^{\prime}\right)=\langle v\rangle^{-\beta}\left\langle v^{\prime}\right\rangle^{-\beta}$ with arbitrary $\beta>0$, as well as Boltzmann kernels $\mathrm{b}\left(v, v^{\prime}\right)=\left|v-v^{\prime}\right|^{-\beta}$ with $0<\beta<d$.

As a consequence of (H2) the collision frequency

$$
\nu_{2}(v)=\int_{\mathbb{R}^{d}} \mathrm{~b}\left(v, v^{\prime}\right) F\left(v^{\prime}\right) \mathrm{d} v^{\prime}
$$


satisfies

$$
\begin{aligned}
\underline{\mathrm{b}}\langle v\rangle^{-\beta} \int_{\mathbb{R}^{d}}\left\langle v^{\prime}\right\rangle^{-\beta} F\left(v^{\prime}\right) \mathrm{d} v^{\prime} \leq \nu_{2}(v) \leq & \overline{\mathrm{b}} \int_{\left|v-v^{\prime}\right|<1}\left|v-v^{\prime}\right|^{-\gamma} F\left(v^{\prime}\right) \mathrm{d} v^{\prime} \\
& +\overline{\mathrm{b}} \int_{\left|v-v^{\prime}\right|>1}\left|v-v^{\prime}\right|^{-\beta} F\left(v^{\prime}\right) \mathrm{d} v^{\prime} .
\end{aligned}
$$

It is obvious that the last term is $O\left(|v|^{-\beta}\right)$ as $|v| \rightarrow \infty$, and the first term on the right hand side is asymptotically small compared to that as a consequence of the sub-exponential decay of $F$. Therefore there exist constants $\bar{\nu} \geq \underline{\nu}>0$ such that

$$
\underline{\nu}\langle v\rangle^{-\beta} \leq \nu_{2}(v) \leq \bar{\nu}\langle v\rangle^{-\beta} \quad \forall v \in \mathbb{R}^{d},
$$

and the behavior for large $|v|$ is as in (3) with $\beta=2(1-\alpha)$.

Since both collision operators are propagators of Markov processes with the same positive stationary distribution $F$, they also share the (quadratic) entropy dissipation property

$$
\frac{1}{2} \frac{\mathrm{d}}{\mathrm{d} t} \iint_{\mathbb{R}^{d} \times \mathbb{R}^{d}} f^{2} \mathrm{~d} x \mathrm{~d} \mu=\iint_{\mathbb{R}^{d} \times \mathbb{R}^{d}}(\mathrm{~L} f) f \mathrm{~d} x \mathrm{~d} \mu \leq 0, \quad \text { with } \mathrm{d} \mu(v):=\frac{\mathrm{d} v}{F(v)},
$$

where the dissipations are given by

$$
-\int_{\mathbb{R}^{d}}\left(\mathrm{~L}_{1} f\right) f \mathrm{~d} \mu=\int_{\mathbb{R}^{d}}\left|\nabla_{v} \frac{f}{F}\right|^{2} F \mathrm{~d} v
$$

and

$$
-\int_{\mathbb{R}^{d}}\left(\mathrm{~L}_{2} f\right) f \mathrm{~d} \mu=\frac{1}{2} \int_{\mathbb{R}^{d}} \int_{\mathbb{R}^{d}} \mathrm{~b}\left(v, v^{\prime}\right)\left(f^{\prime} F-f F^{\prime}\right)^{2} \mathrm{~d} \mu \mathrm{d} \mu^{\prime}
$$

with the prime denoting evaluation at $v^{\prime}$. For a derivation of $(6)$ see, e.g., $[7,17]$.

Our purpose is to consider solutions of (1) with non-negative initial datum $f^{\text {in }}$ and to study their large time behavior. If $f^{\text {in }}$ has finite mass, then mass is conserved for any $t \geq 0$. Since there is no stationary state with finite mass, it is expected that $f(t, \cdot, \cdot)$ locally tends to zero as $t \rightarrow+\infty$. However, the dissipations (5) and (6) vanish for arbitrary local equilibria of the form $f(t, x, v)=\rho(t, x) F(v)$, and therefore the analysis of the decay to zero requires an hypocoercivity method.

For the formulation of our main result, we introduce the norms

$$
\|f\|_{k}:=\left(\iint_{\mathbb{R}^{d} \times \mathbb{R}^{d}} f^{2}\langle v\rangle^{k} \mathrm{~d} x \mathrm{~d} \mu\right)^{1 / 2}, \quad k \in \mathbb{R},
$$

as well as the scalar product $\left\langle f_{1}, f_{2}\right\rangle:=\iint_{\mathbb{R}^{d} \times \mathbb{R}^{d}} f_{1} f_{2} \mathrm{~d} x \mathrm{~d} \mu$ on $\mathrm{L}^{2}(\mathrm{~d} x \mathrm{~d} \mu)$ with the induced norm $\|f\|^{2}:=\|f\|_{0}^{2}=\langle f, f\rangle$.

Theorem 1. Let $\alpha \in(0,1), \beta>0, k>0$ and let $F$ be given by (2). Assume that either $\mathrm{L}=\mathrm{L}_{1}$ and $\beta=2(1-\alpha)$, or $\mathrm{L}=\mathrm{L}_{2}$ and $(\mathrm{H} 1),(\mathrm{H} 2)$. Then there exists a constant $\mathcal{C}>0$ such that any solution $f$ of (1) with initial datum $f^{\text {in }} \in \mathrm{L}^{2}\left(\langle v\rangle^{k} \mathrm{~d} x \mathrm{~d} \mu\right) \cap \mathrm{L}_{+}^{1}(\mathrm{~d} x \mathrm{~d} v)$ 
satisfies

$$
\|f(t, \cdot, \cdot)\|^{2} \leq \mathcal{C} \frac{\left\|f^{\text {in }}\right\|^{2}}{(1+\kappa t)^{\zeta}} \quad t \geq 0
$$

with rate $\zeta=\min \{d / 2, k / \beta\}$ and with $\kappa>0$, which is an explicit function of the two quotients $\left\|f^{\text {in }}\right\| /\left\|f^{\text {in }}\right\|_{k}$ and $\left\|f^{\text {in }}\right\| /\left\|f^{\text {in }}\right\|_{\mathrm{L}^{1}(\mathrm{~d} x \mathrm{~d} v)}$.

The proof relies on the $\mathrm{L}^{2}$-hypocoercivity approach of $[9,10]$. An important ingredient is microscopic coercivity, meaning that the entropy dissipation controls the distance to the set of local equilibria. For $L=L_{1}$ and for the exponential and superexponential cases $\alpha \geq 1$, this control is provided by the Poincaré inequality

$$
\int_{\mathbb{R}^{d}}\left|\nabla_{v} g\right|^{2} F \mathrm{~d} v \geq \mathcal{C}_{P} \int_{\mathbb{R}^{d}}(g-\bar{g})^{2} F \mathrm{~d} v,
$$

with $\bar{g}=\int_{\mathbb{R}^{d}} g F \mathrm{~d} v$ and $\mathcal{C}_{P}>0$ implying, with $g=f / F$,

$$
-\left\langle\mathrm{L}_{1} f, f\right\rangle \geq \mathcal{C}_{P}\left\|f-\rho_{f} F\right\|^{2},
$$

with $\rho_{f}=\int_{\mathbb{R}^{d}} f d v$. For this case the result of Theorem 1 (with $k=0$ ) has been proven in [4]. For the sub-exponential case of this work we shall prove a relaxed version.

Lemma 2. Let $F$ be given by (2) with $0<\alpha<1$. Let either $\mathrm{L}=\mathrm{L}_{1}$ and $\beta=2(1-\alpha)$ or $\mathrm{L}=\mathrm{L}_{2}$ assuming $(\mathrm{H} 1)$, (H2). Then there exists $\mathcal{C}>0$ such that

$$
-\langle\mathrm{L} f, f\rangle \geq \mathcal{C}\left\|f-\rho_{f} F\right\|_{-\beta}^{2} \quad \forall f \in \mathcal{D}\left(\mathbb{R}^{2 d}\right) .
$$

Proof. For $\mathrm{L}=\mathrm{L}_{1}$ the result is a consequence of the weighted Poincaré inequality

$$
\int_{\mathbb{R}^{d}}\left|\nabla_{v} g\right|^{2} F \mathrm{~d} \mu \geq \mathcal{C} \int_{\mathbb{R}^{d}}(g-\bar{g})^{2}\langle v\rangle^{-2(1-\alpha)} F \mathrm{~d} v \quad \forall g \in \mathcal{D}\left(\mathbb{R}^{d}\right),
$$

which will be proved in Appendix A.

For $\mathrm{L}=\mathrm{L}_{2}$ we estimate

$$
\begin{aligned}
& \int_{\mathbb{R}^{d}}\left(f-\rho_{f} F\right)^{2}\langle v\rangle^{-\beta} \mathrm{d} \mu=\int_{\mathbb{R}^{d}}\left(\int_{\mathbb{R}^{d}}\left(f F^{\prime}-f^{\prime} F\right) F^{\prime} \mathrm{d} \mu^{\prime}\right)^{2}\langle v\rangle^{-\beta} \mathrm{d} \mu \\
& \leq \int_{\mathbb{R}^{d}} F\langle v\rangle^{\beta} \mathrm{d} v \iint_{\mathbb{R}^{d} \times \mathbb{R}^{d}}\left(f F^{\prime}-f^{\prime} F\right)^{2}\langle v\rangle^{-\beta}\left\langle v^{\prime}\right\rangle^{-\beta} \mathrm{d} \mu \mathrm{d} \mu^{\prime} \leq-\frac{1}{\mathcal{C}} \int_{\mathbb{R}^{d}}\left(\mathrm{~L}_{2} f\right) f \mathrm{~d} \mu
\end{aligned}
$$

with

$$
\mathcal{C}=\frac{\underline{\mathrm{b}}}{2}\left(\int_{\mathbb{R}^{d}} F\langle v\rangle^{\beta} \mathrm{d} v\right)^{-1} .
$$

For the first inequality we have used Cauchy-Schwarz and for the second, (6) and the hypothesis (H2). Integration with respect to $x$ completes the proof.

Apart from proving the weighted Poincaré inequality (9) (in Appendix A), we shall also show (in Appendix B) how it can be used to prove algebraic decay to equilibrium for the spatially homogeneous equation with $\mathrm{L}=\mathrm{L}_{1}$ and $0<\alpha<1$, i.e. the FokkerPlanck equation with sub-exponential equilibrium. The loss of information due to the 
weight $\langle v\rangle^{-2(1-\alpha)}$ has to be compensated by a $\mathrm{L}^{2}$-bound for the initial datum with a weight $\langle v\rangle^{k}, k>0$, as in Theorem 1. For this problem, estimates based on weak Poincaré inequalities are also very popular in the scientific community of semi-group theory and Markov processes (see [22], [3, Proposition 7.5.10], [14] and Appendix B). Estimates based on weak Poincaré inequalities rely on a uniform bound for the initial data for $\alpha<1$ which is not needed for $\alpha \geq 1$, while the approach developed in this paper provides a continuous transition from the range $0<\alpha<1$ to the range $\alpha \geq 1$ since we may choose $k \searrow 0$ as $\alpha \nearrow 1$. Note that for $\alpha=1$, the weighted Poincaré inequality (9) reduces to the Poincaré inequality (8).

The proof of Theorem 1 follows along the lines of the hypocoercivity approach of $[9$, 10] and its extension to cases without confinement as in [4, 5]. It combines information on the microscopic and the macroscopic dissipation properties. The essence of the microscopic information is given in Lemma 2. Since the macroscopic limit of (1) is the heat equation on the whole space, it is natural that for the estimation of the macroscopic dissipation we use Nash's inequality, a tool which has been developed for this purpose. The result of Theorem 1 can be interpreted as giving the weaker of the microscopic decay rate $t^{-k / \beta}$ and the macroscopic decay rate $t^{-d / 2}$. Only for $k \geq \beta d / 2$ the decay rate of the macroscopic diffusion limit is recovered.

Related results have been shown in $[4,5,6]$, where the latter is somewhat complementary to this work dealing with Gaussian local equilibria in the presence of an external potential with sub-exponential growth in the variable $x$. Also see $[24,15,13]$ for various earlier results dealing with external potentials with a growth like $\langle x\rangle^{\gamma}$, $\gamma<1$, based on weak Poincaré inequalities, spectral techniques, $\mathrm{H}^{1}$ hypocoercivity methods, etc.

This paper is organized as follows. In Section 2, we prove an hypocoercive estimate relating a modified entropy, which is equivalent to $\|f\|^{2}$, to an entropy production term involving a microscopic and a macroscopic component. Using weighted $\mathrm{L}^{2}$-estimates established in Section 3, we obtain a new control by the microscopic component in Lemma 8 while the macroscopic component is estimated as in [4] using Nash's inequality, see Lemma 7. By collecting these estimates in Section 4, we complete the proof of Theorem 1. Two appendices are devoted to $L=L_{1}$ : in Appendix $A$ we provide a new proof of (9) and comment on the interplay with weak Poincaré inequalities, while the spatially homogeneous version of (1) is dealt with in Appendix B and rates of relaxation towards the local equilibrium are discussed using weighted $\mathrm{L}^{2}$-norms, as an alternative approach to the weak Poincaré inequality method of [14]. The main novelty of our approach is that we use new interpolations in order to exploit the entropy production term. As a consequence, with the appropriate weights, no other norm is needed than weighted $\mathrm{L}^{2}$-norms. For simplicity, we assume that the distribution function is nonnegative but the extension to sign changing functions is straightforward. 


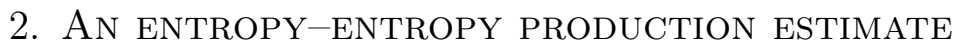

We adapt the strategy of $[10,4]$, denoting by $\mathrm{T}=v \cdot \nabla_{x}$ the free streaming operator and by $\Pi$ the orthogonal projection on $\operatorname{Ker}(L)$ in $L^{2}\left(\mathbb{R}^{d}, \mathrm{~d} \mu\right)$, given by

$$
\Pi f:=\rho_{f} F \quad \text { where } \rho_{f}=\int_{\mathbb{R}^{d}} f \mathrm{~d} v .
$$

To build a suitable Lyapunov functional, we introduce the operator

$$
\mathrm{A}:=\left(\mathrm{Id}+(\mathrm{T \Pi})^{*}(\mathrm{~T} \Pi)\right)^{-1}(\mathrm{~T} \Pi)^{*}
$$

and consider

$$
\mathrm{H}[f]:=\frac{1}{2}\|f\|^{2}+\delta\langle\mathrm{A} f, f\rangle .
$$

It is known from [10, Lemma 1] that, for any $\delta \in(0,1), \mathrm{H}[f]$ and $\|f\|^{2}$ are equivalent in the sense that

$$
\frac{1}{2}(1-\delta)\|f\|^{2} \leq \mathrm{H}[f] \leq \frac{1}{2}(1+\delta)\|f\|^{2} .
$$

A direct computation shows that

$$
\frac{\mathrm{d}}{\mathrm{d} t} \mathrm{H}[f]=-\mathrm{D}[f]
$$

with

$$
\begin{aligned}
\mathrm{D}[f]:= & -\langle\mathrm{L} f, f\rangle+\delta\langle\mathrm{AT} \Pi f, \Pi f\rangle \\
& +\delta\langle\mathrm{AT}(\mathrm{Id}-\Pi) f, \Pi f\rangle-\delta\langle\mathrm{TA}(\mathrm{Id}-\Pi) f,(\mathrm{Id}-\Pi) f\rangle-\delta\langle\mathrm{AL}(\mathrm{Id}-\Pi) f, \Pi f\rangle
\end{aligned}
$$

where we have used that $\langle\mathrm{A} f, \mathrm{~L} f\rangle=0$. Note that in terms of the new notation the result of Lemma 2 reads

$$
\langle\mathrm{L} f, f\rangle \leq-\mathcal{C}\|(\mathrm{Id}-\Pi) f\|_{-\beta}^{2} .
$$

Proposition 3. Under the assumptions of Theorem 1 and for small enough $\delta>0$, there exists $\kappa>0$ such that, for any $f \in \mathrm{L}^{2}\left(\langle v\rangle^{-\beta} \mathrm{d} x \mathrm{~d} \mu\right) \cap \mathrm{L}^{1}(\mathrm{~d} x \mathrm{~d} v)$,

$$
\mathrm{D}[f] \geq \kappa\left(\|(\mathrm{Id}-\Pi) f\|_{-\beta}^{2}+\langle\operatorname{AT} \Pi f, \Pi f\rangle\right) .
$$

Note that $\kappa$ does not depend on $k>0$ (the parameter $k$ appears in the assumptions of Theorem 1). An estimate of $\mathrm{D}[f]$ in terms of $\langle$ AT $\Pi f, \Pi f\rangle$ and $\|(\mathrm{Id}-\Pi) f\|^{2}$ has already been derived in [4], but using the weighted norm $\|(\mathrm{Id}-\Pi) f\|_{-\beta}$ is a new idea.

Proof. We have to prove that the three last terms in $\mathrm{D}[f]$ are controlled by the first two. The main difference with $[10,4]$ is the additional weight $\langle v\rangle^{-\beta}$ in the velocity variable.

- Step 1: rewriting $\langle$ АТП $f, \Pi f\rangle$. Let $u=u_{f}$ be such that

$$
u F=\left(\mathrm{Id}+(\mathrm{T} \Pi)^{*}(\mathrm{~T} \Pi)\right)^{-1} \Pi f .
$$


Then $u$ solves $(u-\Theta \Delta u) F=\Pi f$, that is,

$$
u-\Theta \Delta u=\rho_{f}
$$

where $\Theta:=\int_{\mathbb{R}^{d}}|v \cdot \mathrm{e}|^{2} F(v) \mathrm{d} v$ for an arbitrary unit vector e. Since

$$
\begin{aligned}
\text { AT } f & =\left(\mathrm{Id}+(\mathrm{T} \Pi)^{*}(\mathrm{~T} \Pi)\right)^{-1}(\mathrm{~T} \Pi)^{*}(\mathrm{~T} \Pi) \Pi f \\
& =\left(\mathrm{Id}+(\mathrm{T} \Pi)^{*}(\mathrm{~T} \Pi)\right)^{-1}\left(\mathrm{Id}+(\mathrm{T} \Pi)^{*}(\mathrm{~T} \Pi)-\mathrm{Id}\right) \Pi f \\
& =\Pi f-\left(\mathrm{Id}+(\mathrm{T} \Pi)^{*}(\mathrm{~T} \Pi)\right)^{-1} \Pi f=\Pi f-u F=\left(\rho_{f}-u\right) F,
\end{aligned}
$$

then by using equation (13), we obtain

$$
\langle\operatorname{AT} \Pi f, \Pi f\rangle=\langle\Pi f-u F, \Pi f\rangle=\langle-\Theta \Delta u F,(u-\Theta \Delta u) F\rangle,
$$

from which we deduce

$$
\langle\operatorname{AT\Pi } f, \Pi f\rangle=\Theta\|\nabla u\|_{\mathrm{L}^{2}(\mathrm{~d} x)}^{2}+\Theta^{2}\|\Delta u\|_{\mathrm{L}^{2}(\mathrm{~d} x)}^{2} \geq 0 .
$$

- Step 2: a bound on $\langle$ AT(Id $-\Pi) f$, $\Pi f\rangle$. If $u$ solves (13), we use the fact that

$$
\mathrm{A}^{*} \Pi f=\mathrm{T} \Pi u F=\mathrm{T} u F
$$

to compute

$$
\langle\mathrm{AT}(\mathrm{Id}-\Pi) f, \Pi f\rangle=\left\langle(\mathrm{Id}-\Pi) f, \mathrm{~T}^{*} \mathrm{~A}^{*} \Pi f\right\rangle=\left\langle(\mathrm{Id}-\Pi) f, \mathrm{~T}^{*} \mathrm{~T} u F\right\rangle .
$$

Therefore, since $\mathrm{T}^{*} \mathrm{~T} u F=-v \cdot \nabla_{x}\left(v \cdot \nabla_{x} u\right) F$, the Cauchy-Schwarz inequality yields

$$
\begin{array}{r}
|\langle\mathrm{AT}(\mathrm{Id}-\Pi) f, \Pi f\rangle| \leq\|(\mathrm{Id}-\Pi) f\|_{-\beta}\left\|\sqrt{F}\langle v\rangle^{\frac{\beta}{2}} v \cdot \nabla_{x}\left(v \cdot \nabla_{x} u\right) \sqrt{F}\right\|_{\mathrm{L}^{2}(\mathrm{~d} x \mathrm{~d} v)} \\
\leq \Theta_{\beta+4}\|(\mathrm{Id}-\Pi) f\|_{-\beta}\|\Delta u\|_{\mathrm{L}^{2}(\mathrm{~d} x)}
\end{array}
$$

hence

$$
|\langle\mathrm{AT}(\mathrm{Id}-\Pi) f, \Pi f\rangle| \leq \mathcal{C}_{4}\|(\mathrm{Id}-\Pi) f\|_{-\beta}\langle\operatorname{AT} \Pi f, \Pi f\rangle^{\frac{1}{2}}
$$

where we have used identity $(14), \mathcal{C}_{4}=\Theta_{\beta+4} / \Theta$ and

$$
\Theta_{k}:=\int_{\mathbb{R}^{d}}\langle v\rangle^{k} F(v) \mathrm{d} v .
$$

With this convention, note that $\Theta_{2}=1+d \Theta$.

- Step 3: estimating $\langle\mathrm{TA}(\mathrm{Id}-\Pi) f,(\mathrm{Id}-\Pi) f\rangle$. As noted in [10, Lemma 1], the equation $g=\Pi g=$ A $f$ is equivalent to

$$
\left(\mathrm{Id}+(\mathrm{T} \Pi)^{*}(\mathrm{~T} \Pi)\right) g=(\mathrm{T} \Pi)^{*} f
$$

which, after multiplying by $g$ and integrating, yields

$$
\begin{aligned}
\|g\|^{2}+\|\mathrm{T} g\|^{2} & =\left\langle g, g+(\mathrm{T} \Pi)^{*}(\mathrm{~T} \Pi) g\right\rangle \\
& =\left\langle g,(\mathrm{~T} \Pi)^{*} f\right\rangle=\langle\mathrm{T} \Pi g, f\rangle=\langle\mathrm{TA} f, f\rangle \leq\|(\mathrm{Id}-\Pi) f\|_{-\beta}\|\mathrm{TA} f\|_{\beta}
\end{aligned}
$$


by the Cauchy-Schwarz inequality. We know that $(\mathrm{T} \Pi)^{*}=-\Pi \mathrm{T}$ so that $\mathrm{A} f=g=w F$ is determined by the equation

$$
w-\Theta \Delta w=-\nabla_{x} \cdot \int_{\mathbb{R}^{d}} v f \mathrm{~d} v
$$

After multiplying by $w$ and integrating in $x$, we obtain that

$$
\begin{aligned}
\Theta \int_{\mathbb{R}^{d}}\left|\nabla_{x} w\right|^{2} \mathrm{~d} x \leq \int_{\mathbb{R}^{d}}|w|^{2} \mathrm{~d} x+\Theta \int_{\mathbb{R}^{d}} & \left|\nabla_{x} w\right|^{2} \mathrm{~d} x \\
& \leq\left(\int_{\mathbb{R}^{d}}\left|\nabla_{x} w\right|^{2} \mathrm{~d} x\right)^{\frac{1}{2}}\left(\int_{\mathbb{R}^{d}}\left|\int_{\mathbb{R}^{d}} v f \mathrm{~d} v\right|^{2} \mathrm{~d} x\right)^{\frac{1}{2}}
\end{aligned}
$$

and note that

$$
\begin{aligned}
\int_{\mathbb{R}^{d}}\left|\int_{\mathbb{R}^{d}} v f \mathrm{~d} v\right|^{2} \mathrm{~d} x=\int_{\mathbb{R}^{d}}\left|\int_{\mathbb{R}^{d}}\langle v\rangle^{-\frac{\beta}{2}} \frac{(\mathrm{Id}-\Pi) f}{\sqrt{F}} \cdot\right| v\left|\langle v\rangle^{\frac{\beta}{2}} \sqrt{F} \mathrm{~d} v\right|^{2} \mathrm{~d} x & \leq \Theta_{\beta+2}\|(\mathrm{Id}-\Pi) f\|_{-\beta}^{2}
\end{aligned}
$$

by the Cauchy-Schwarz inequality. Hence

$$
\int_{\mathbb{R}^{d}}\left|\nabla_{x} w\right|^{2} \mathrm{~d} x \leq \frac{\Theta_{\beta+2}}{\Theta^{2}}\|(\mathrm{Id}-\Pi) f\|_{-\beta}^{2}
$$

and

$$
\|\mathrm{TA} f\|_{\beta}^{2}=\left\|\nabla_{x} w \cdot\left(v\langle v\rangle^{\beta / 2} F\right)\right\|^{2}=\Theta_{\beta+2} \int_{\mathbb{R}^{d}}\left|\nabla_{x} w\right|^{2} \mathrm{~d} x \leq \mathcal{C}_{2}^{2}\|(\mathrm{Id}-\Pi) f\|_{-\beta}^{2}
$$

with $\mathcal{C}_{2}:=\Theta_{\beta+2} / \Theta$. Since $g=\mathrm{A} f$ so that $\|\mathrm{A} f\|^{2}+\|\mathrm{TA} f\|^{2}=\|g\|^{2}+\|\mathrm{T} g\|^{2}$, we obtain that

$$
\langle\mathrm{TA} f, f\rangle=\langle\mathrm{TA}(\mathrm{Id}-\Pi) f,(\mathrm{Id}-\Pi) f\rangle \leq\|(\mathrm{Id}-\Pi) f\|_{-\beta}\|\mathrm{TA} f\|_{\beta} \leq \mathcal{C}_{2}\|(\mathrm{Id}-\Pi) f\|_{-\beta}^{2} .
$$

We also remark that

$$
\begin{aligned}
\langle\mathrm{TA} f, f\rangle=\left\langle\left(v \cdot \nabla_{x} w\right) F, f\right\rangle=\int_{\mathbb{R}^{d}} \nabla_{x} w \cdot\left(\int_{\mathbb{R}^{d}} v f \mathrm{~d} v\right) \mathrm{d} x & \\
& =\int_{\mathbb{R}^{d}}|w|^{2} \mathrm{~d} x+\Theta \int_{\mathbb{R}^{d}}\left|\nabla_{x} w\right|^{2} \mathrm{~d} x \geq 0 .
\end{aligned}
$$

- Step 4: bound for $\langle\mathrm{AL}(\mathrm{Id}-\Pi) f, \Pi f\rangle$. We use again identity (15) to compute

$$
\begin{aligned}
|\langle\operatorname{AL}(\mathrm{Id}-\Pi) f, \Pi f\rangle|=\left|\left\langle(\mathrm{Id}-\Pi) f, \mathrm{~L}^{*} \mathrm{~A}^{*} \Pi f\right\rangle\right|=\left|\left\langle(\mathrm{Id}-\Pi) f, \mathrm{~L}^{*} \mathrm{~T} u F\right\rangle\right| \\
\quad \leq\|(\mathrm{Id}-\Pi) f\|_{-\beta}\left\|\mathrm{L}^{*} \mathrm{~T} u F\right\|_{\beta} .
\end{aligned}
$$

In case $\mathrm{L}=\mathrm{L}_{1}$ we remark that

$$
\begin{aligned}
\left\|\mathrm{L}_{1}^{*} \mathrm{~T} u F\right\|_{\beta}^{2}= & \iint_{\mathbb{R}^{d} \times \mathbb{R}^{d}}\left|\nabla_{v} \cdot\left(F \nabla_{v}\left(v \cdot \nabla_{x} u\right)\right)\right|^{2}\langle v\rangle^{\beta} \mathrm{d} x \mathrm{~d} \mu \\
& =\iint_{\mathbb{R}^{d} \times \mathbb{R}^{d}}\left|\nabla_{v} F \cdot \nabla_{x} u\right|^{2}\langle v\rangle^{\beta} \mathrm{d} x \mathrm{~d} \mu \leq\left\|\nabla_{v} F\right\|_{\mathrm{L}^{2}\left(\langle v\rangle^{\beta} \mathrm{d} \mu\right)}^{2}\left\|\nabla_{x} u\right\|_{\mathrm{L}^{2}(\mathrm{~d} x)}^{2} .
\end{aligned}
$$


In case $\mathrm{L}=\mathrm{L}_{2}$, note first that

$$
\left(\mathrm{L}_{2}^{*} \mathrm{~T} u F\right)(v)=\left(\int_{\mathbb{R}^{d}} \mathrm{~b}\left(v^{\prime}, v\right)\left(v^{\prime}-v\right) F\left(v^{\prime}\right) \mathrm{d} v^{\prime}\right) \cdot \nabla_{x} u F(v),
$$

and thus, by the Cauchy-Schwarz inequality,

$$
\left\|\mathrm{L}_{2}^{*} \mathrm{~T} u F\right\|_{\beta} \leq \mathcal{B}\left\|\nabla_{x} u\right\|_{\mathrm{L}^{2}(\mathrm{~d} x)}, \quad \text { with } \quad \mathcal{B}=\left\|\int_{\mathbb{R}^{d}} \mathrm{~b}\left(v^{\prime}, v\right)\left(v^{\prime}-v\right) F^{\prime} F \mathrm{~d} v^{\prime}\right\|_{\mathrm{L}^{2}\left(\langle v\rangle^{\beta} \mathrm{d} \mu\right)} .
$$

For proving finiteness of $\mathcal{B}$ we use $(\mathrm{H} 2)$ in

$$
\begin{aligned}
& \left|\int_{\mathbb{R}^{d}} \mathrm{~b}\left(v^{\prime}, v\right)\left(v^{\prime}-v\right) F^{\prime} \mathrm{d} v^{\prime}\right| \\
& \quad \leq \overline{\mathrm{b}} \int_{\left|v^{\prime}-v\right|<1}\left|v^{\prime}-v\right|^{1-\gamma} F^{\prime} \mathrm{d} v^{\prime}+\overline{\mathrm{b}} \int_{\left|v^{\prime}-v\right|>1}\left|v^{\prime}-v\right|^{1-\beta} F^{\prime} \mathrm{d} v^{\prime} \leq c\left(1+\langle v\rangle^{1-\beta}\right),
\end{aligned}
$$

which implies

$$
\mathcal{B}^{2} \leq c^{2} \int_{\mathbb{R}^{d}}\left(1+\langle v\rangle^{1-\beta}\right)^{2}\langle v\rangle^{\beta} F \mathrm{~d} v<\infty
$$

Combining these estimates with identity (14) we get

$$
|\langle\operatorname{AL}(\mathrm{Id}-\Pi) f, \Pi f\rangle| \leq \mathcal{C}_{F}\|(\mathrm{Id}-\Pi) f\|_{-\beta}\langle\operatorname{AT} \Pi f, \Pi f\rangle^{\frac{1}{2}}
$$

where $\mathcal{C}_{F}=\mathcal{B} / \sqrt{\Theta}$.

- Step 5: collecting all estimates. Altogether, combining (12) and (16), (17) and (18), we obtain

$$
\begin{aligned}
\frac{\mathrm{d}}{\mathrm{d} t} \mathrm{H}[f] \leq-\mathcal{C} \|(\mathrm{Id} & -\Pi) f \|_{-\beta}^{2}-\delta\langle\operatorname{AT} \Pi f, \Pi f\rangle \\
& +\delta\left(\mathcal{C}_{4}+\mathcal{C}_{F}\right)\|(\mathrm{Id}-\Pi) f\|_{-\beta}\langle\operatorname{AT} \Pi f, \Pi f\rangle^{\frac{1}{2}}+\delta \mathcal{C}_{2}\|(\mathrm{Id}-\Pi) f\|_{-\beta}^{2}
\end{aligned}
$$

which by Young's inequality yields the existence of $\kappa>0$ such that

$$
\frac{\mathrm{d}}{\mathrm{d} t} \mathrm{H}[f] \leq-\kappa\left(\|(\mathrm{Id}-\Pi) f\|_{-\beta}^{2}+\langle\operatorname{AT\Pi } f, \Pi f\rangle\right)
$$

for some $\delta \in(0,1)$. Indeed, with $X:=\|(\operatorname{Id}-\Pi) f\|_{-\beta}$ and $Y:=\langle\operatorname{AT} \Pi f, \Pi f\rangle^{\frac{1}{2}}$, it is enough to check that the quadratic form

$$
\mathcal{Q}(X, Y):=\left(\mathcal{C}-\delta \mathcal{C}_{2}\right) X^{2}-\left(\mathcal{C}_{4}+\mathcal{C}_{F}\right) X Y+\delta Y^{2}
$$

is positive, i.e., $\mathcal{Q}(X, Y) \geq \kappa\left(X^{2}+Y^{2}\right)$ for some $\kappa=\kappa(\delta)$ and $\delta \in(0,1)$.

\section{Weighted L ${ }^{2}$ estimates}

In this section, we show the propagation of weighted norms with weights $\langle v\rangle^{k}$ of arbitrary positive order $k \in \mathbb{R}^{+}$. 
Proposition 4. Let $k>0$ and $f$ be solution of (1) with $f^{\text {in }} \in \mathrm{L}^{2}\left(\langle v\rangle^{k} \mathrm{~d} x \mathrm{~d} \mu\right)$. Then there exists a constant $\mathcal{K}_{k}>1$ such that

$$
\forall t \geq 0 \quad\|f(t, \cdot, \cdot)\|_{k} \leq \mathcal{K}_{k}\left\|f^{\text {in }}\right\|_{k} .
$$

We recall that $\|f\|_{k}$ is defined by (7). We shall state a technical lemma (Lemma 5 below) before proving a splitting result in Lemma 6, from which the proof of Proposition 4 easily follows (see section 3.3).

\subsection{A technical lemma.}

Lemma 5. If either $\mathrm{L}=\mathrm{L}_{1}$ or $\mathrm{L}=\mathrm{L}_{2}$, then there exists $\ell>0$ for which, for any $k \geq 0$, there exist $a_{k}, b_{k}, R_{k}>0$ such that

$$
\left\langle\mathrm{L} f, f\langle v\rangle^{k}\right\rangle \leq \iint_{\mathbb{R}^{d} \times \mathbb{R}^{d}}\left(a_{k} \mathbb{1}_{|v|<R_{k}}-b_{k}\langle v\rangle^{-\ell}\right)|f|^{2}\langle v\rangle^{k} \mathrm{~d} x \mathrm{~d} \mu,
$$

for any $f \in \mathcal{D}\left(\mathbb{R}^{2 d}\right)$.

Proof. In the Fokker-Planck case $\mathrm{L}=\mathrm{L}_{1}$ we have

$$
\begin{aligned}
& \int_{\mathbb{R}^{d}} \mathrm{~L}_{1} f f\langle v\rangle^{k} \mathrm{~d} \mu=-\int_{\mathbb{R}^{d}}\left|\nabla_{v}\left(\frac{f}{F}\right)\right|^{2}\langle v\rangle^{k} F \mathrm{~d} v-\frac{1}{2} \int_{\mathbb{R}^{d}} \nabla_{v}\left(\frac{f^{2}}{F^{2}}\right) \cdot \nabla_{v}\langle v\rangle^{k} F \mathrm{~d} \mu \\
& \leq \frac{1}{2} \int_{\mathbb{R}^{d}} f^{2}\left(\frac{\nabla_{v} F}{F} \cdot \nabla_{v}\langle v\rangle^{k}+\Delta_{v}\langle v\rangle^{k}\right) \mathrm{d} \mu \\
&= \frac{k}{2} \int_{\mathbb{R}^{d}} f^{2}\langle v\rangle^{k-4}\left(2-k+(d+k-2)\langle v\rangle^{2}+\alpha\langle v\rangle^{\alpha}-\alpha\langle v\rangle^{\alpha+2}\right) \mathrm{d} \mu \\
& \leq \frac{k}{2} \int_{\mathbb{R}^{d}} f^{2}\langle v\rangle^{k-2}\left(c_{k}-\alpha\langle v\rangle^{\alpha}\right) \mathrm{d} \mu \\
&= \int_{\mathbb{R}^{d}}\left(a_{k} \mathbb{1}_{|v|<R_{k}}-b_{k}\langle v\rangle^{-\ell}\right)|f|^{2}\langle v\rangle^{k} \mathrm{~d} \mu \\
& \quad+\frac{k}{2} \int_{\mathbb{R}^{d}} f^{2}\langle v\rangle^{k-2}\left(c_{k}\left(1-\mathbb{1}_{|v|<R_{k}}\langle v\rangle^{2}\right)-\frac{\alpha}{2}\langle v\rangle^{\alpha}\right) \mathrm{d} \mu,
\end{aligned}
$$

with $c_{k}=|k-2|+|d+k-2|+\alpha, a_{k}=c_{k} k / 2, b_{k}=\alpha k / 4, \ell=2-\alpha$. The choice $R_{k}=\left(2 c_{k} / \alpha\right)^{1 / \alpha}$ makes the last term negative, which completes the proof.

In the case of the scattering operator $\mathrm{L}=\mathrm{L}_{2}$, with $h:=f / F$, we have

$$
\begin{aligned}
& 2 \int_{\mathbb{R}^{d}} f \mathrm{~L}_{2} f\langle v\rangle^{k} \mathrm{~d} \mu=2 \iint_{\mathbb{R}^{d} \times \mathbb{R}^{d}} \mathrm{~b}\left(v, v^{\prime}\right)\left(h^{\prime}-h\right) h\langle v\rangle^{k} F F^{\prime} \mathrm{d} v \mathrm{~d} v^{\prime} \\
&=\iint_{\mathbb{R}^{d} \times \mathbb{R}^{d}} \mathrm{~b}\left(v, v^{\prime}\right)\left(2 h^{\prime} h-h^{2}\right)\langle v\rangle^{k} F F^{\prime} \mathrm{d} v \mathrm{~d} v^{\prime} \\
&-\iint_{\mathbb{R}^{d} \times \mathbb{R}^{d}} \mathrm{~b}\left(v^{\prime}, v\right) h^{2}\langle v\rangle^{k} F F^{\prime} \mathrm{d} v \mathrm{~d} v^{\prime},
\end{aligned}
$$


where we have used (H1). Swapping $v$ and $v^{\prime}$ in the last integral gives

$$
\begin{aligned}
2 \int_{\mathbb{R}^{d}} f \mathrm{~L}_{2} f\langle v\rangle^{k} \mathrm{~d} \mu= & -\iint_{\mathbb{R}^{d} \times \mathbb{R}^{d}} \mathrm{~b}\left(v, v^{\prime}\right)\left(h-h^{\prime}\right)^{2}\langle v\rangle^{k} F F^{\prime} \mathrm{d} v \mathrm{~d} v^{\prime} \\
& +\iint_{\mathbb{R}^{d} \times \mathbb{R}^{d}} \mathrm{~b}\left(v, v^{\prime}\right)\left(h^{\prime}\right)^{2}\left(\langle v\rangle^{k}-\left\langle v^{\prime}\right\rangle^{k}\right) F F^{\prime} \mathrm{d} v \mathrm{~d} v^{\prime} \\
\leq & \int_{\mathbb{R}^{d}}\left(\int_{\mathbb{R}^{d}} \mathrm{~b}\left(v^{\prime}, v\right)\left(\left\langle v^{\prime}\right\rangle^{k}-\langle v\rangle^{k}\right) F^{\prime} \mathrm{d} v^{\prime}\right) f^{2} \mathrm{~d} \mu,
\end{aligned}
$$

with another swap $v \leftrightarrow v^{\prime}$ in the last step. Now we employ (H2) and its consequence (4):

$$
\begin{aligned}
\int_{\mathbb{R}^{d}} \mathrm{~b}\left(v^{\prime}, v\right)\left(\left\langle v^{\prime}\right\rangle^{k}-\langle v\rangle^{k}\right) F^{\prime} \mathrm{d} v^{\prime} & =\int_{\mathbb{R}^{d}} \mathrm{~b}\left(v^{\prime}, v\right)\left\langle v^{\prime}\right\rangle^{k} F^{\prime} \mathrm{d} v^{\prime}-\langle v\rangle^{k} \nu_{2}(v) \\
& \leq 2 a_{k}\langle v\rangle^{-\beta}-\underline{\nu}\langle v\rangle^{k-\beta},
\end{aligned}
$$

where the estimation of the first term is analogous to the derivation of (4). This implies

$$
\begin{aligned}
\int_{\mathbb{R}^{d}} \mathrm{~L}_{2} f f\langle v\rangle^{k} \mathrm{~d} \mu \leq & \int_{\mathbb{R}^{d}}\left(a_{k} \mathbb{1}_{|v|<R_{k}}-b_{k}\langle v\rangle^{-\ell}\right)|f|^{2}\langle v\rangle^{k} \mathrm{~d} \mu \\
& +\int_{\mathbb{R}^{d}} f^{2}\langle v\rangle^{k}\left(a_{k}\left(\langle v\rangle^{-\beta-k}-\mathbb{1}_{|v|<R_{k}}\right)+b_{k}\langle v\rangle^{-\ell}-\frac{\nu}{2}\langle v\rangle^{-\beta}\right) \mathrm{d} v .
\end{aligned}
$$

The last term is made negative by the choices $\ell=\beta, b_{k}=\underline{\nu} / 4, R_{k}=\left(4 a_{k} / \underline{\nu}\right)^{1 / k}$.

3.2. A splitting result. As in $[11,14,19]$, we write $\mathrm{L}-\mathrm{T}$ as a dissipative part $\mathrm{C}$ and a bounded part $\mathrm{B}$ such that $\mathrm{L}-\mathrm{T}=\mathrm{B}+\mathrm{C}$.

Lemma 6. With the notation of Lemma 5, let $k_{1}>0, k_{2}>k_{1}+2 \ell, a=\max \left\{a_{k_{1}}, a_{k_{2}}\right\}$, $R=\max \left\{R_{k_{1}}, R_{k_{2}}\right\}, \mathrm{C}=a \mathbb{1}_{|v|<R}$ and $\mathrm{B}=\mathrm{L}-\mathrm{T}-\mathrm{C}$. For any $t \geq 0$ we have:

(i) $\|\mathrm{C}\|_{\mathrm{L}^{2}(\mathrm{~d} x \mathrm{~d} \mu) \rightarrow \mathrm{L}^{2}\left(\langle v\rangle^{k_{2}} \mathrm{~d} x \mathrm{~d} \mu\right)} \leq a\langle R\rangle^{k_{2} / 2}$,

(ii) $\left\|e^{t \mathrm{~B}}\right\|_{\mathrm{L}^{2}\left(\langle v\rangle^{k_{1}} \mathrm{~d} x \mathrm{~d} \mu\right) \rightarrow \mathrm{L}^{2}\left(\langle v\rangle^{k_{1}} \mathrm{~d} x \mathrm{~d} \mu\right)} \leq 1$,

(iii) $\left\|e^{t \mathrm{~B}}\right\|_{\mathrm{L}^{2}\left(\langle v\rangle^{k_{2}} \mathrm{~d} x \mathrm{~d} \mu\right) \rightarrow \mathrm{L}^{2}\left(\langle v\rangle^{k_{1}} \mathrm{~d} x \mathrm{~d} \mu\right)} \leq C(1+t)^{-\frac{k_{2}-k_{1}}{2 \ell}}$ for some $C>0$.

Proof. Property (i) is a consequence of the definition of C. Property (ii) follows from Lemma 5 according to

$$
\begin{aligned}
\iint_{\mathbb{R}^{d} \times \mathbb{R}^{d}} f \mathrm{~B} f\langle v\rangle^{k_{1}} \mathrm{~d} x \mathrm{~d} \mu & \leq \iint_{\mathbb{R}^{d} \times \mathbb{R}^{d}}\left(a_{k_{1}} \mathbb{1}_{B_{R_{k_{1}}}}-a \mathbb{1}_{B_{R}}-b_{k_{1}}\langle v\rangle^{-\ell}\right)|f|^{2}\langle v\rangle^{k_{1}} \mathrm{~d} x \mathrm{~d} \mu \\
& \leq-b_{k_{1}} \iint_{\mathbb{R}^{d} \times \mathbb{R}^{d}}|f|^{2}\langle v\rangle^{k_{1}-\ell} \mathrm{d} x \mathrm{~d} \mu .
\end{aligned}
$$

Similarly, we know that $\left\|e^{t \mathrm{~B}}\right\|_{\mathrm{L}^{2}\left(\langle v\rangle^{k_{2}} \mathrm{~d} x \mathrm{~d} \mu\right) \rightarrow \mathrm{L}^{2}\left(\langle v\rangle^{k_{2}} \mathrm{~d} x \mathrm{~d} \mu\right)} \leq 1$.

By combining Hölder's inequality

$$
\|f\|_{k_{1}}^{2} \leq\|f\|_{k_{1}-\ell}^{\frac{2\left(k_{2}-k_{1}\right)}{k_{2}-k_{1}+\ell}}\|f\|_{k_{2}}^{\frac{2 \ell}{k_{2}-k_{1}+\ell}}
$$


with Property (ii), we obtain

$$
\iint_{\mathbb{R}^{d} \times \mathbb{R}^{d}} f \mathrm{~B} f\langle v\rangle^{k_{1}} \mathrm{~d} x \mathrm{~d} \mu \leq-b_{k_{1}}\|f\|_{k_{1}}^{2\left(1+\frac{\ell}{k_{2}-k_{1}}\right)} \| f^{\text {in } \|_{k_{2}}^{-\frac{2 \ell}{k_{2}-k_{1}}} .}
$$

With $f=e^{t \mathrm{~B}} f^{\text {in }}$, Property (iii) follows from Grönwall's lemma according to

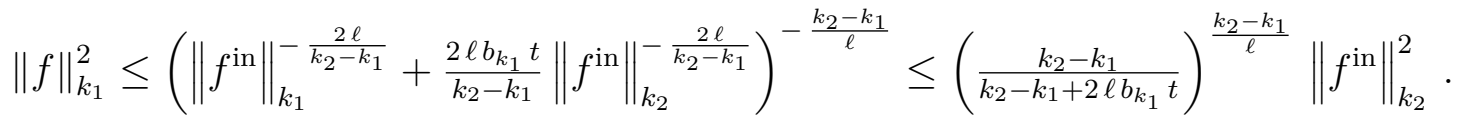

3.3. Proof of Proposition 4. Using the convolution $\mathrm{U} \star \mathrm{V}=\int_{0}^{t} \mathrm{U}(t-s) \mathrm{V}(s) \mathrm{d} s$, Duhamel's formula asserts that

$$
e^{t(\mathbf{L}-\mathbf{T})}=e^{t \mathbf{B}}+e^{t \mathrm{~B}} \star \mathbf{C} e^{t(\mathbf{L}-\mathbf{T})} .
$$

Therefore, by

$$
\left\|e^{t(\mathrm{~L}-\mathrm{T})}\right\|_{\mathrm{L}^{2}(\mathrm{~d} x \mathrm{~d} \mu) \rightarrow \mathrm{L}^{2}(\mathrm{~d} x \mathrm{~d} \mu)} \leq 1 \quad \Rightarrow \quad\left\|e^{t(\mathrm{~L}-\mathrm{T})}\right\|_{\mathrm{L}^{2}\left(\langle v\rangle^{k_{1}} \mathrm{~d} x \mathrm{~d} \mu\right) \rightarrow \mathrm{L}^{2}(\mathrm{~d} x \mathrm{~d} \mu)} \leq 1,
$$

by Lemma 6 , and with $k=k_{1}, \ell$ as in Lemma 5 and $k_{2}>k+2 \ell$, we get that

$$
\left\|e^{t(\mathbf{L}-\mathbf{T})}\right\|_{\mathrm{L}^{2}\left(\langle v\rangle^{k_{1}} \mathrm{~d} x \mathrm{~d} \mu\right) \rightarrow \mathrm{L}^{2}\left(\langle v\rangle^{k_{1}} \mathrm{~d} x \mathrm{~d} \mu\right)} \leq 1+a\langle R\rangle^{\frac{k_{2}}{2}} \int_{0}^{t} \frac{C \mathrm{~d} s}{(1+s)^{\frac{k_{2}-k_{1}}{2 \ell}}}
$$

is bounded uniformly in time.

\section{Proof of Theorem 1}

The control of the macroscopic part $\Pi f$ by $\langle$ AT $\Pi f, \Pi f\rangle$ is achieved as in [4]. We sketch a proof for the sake of completeness.

Lemma 7. Under the assumptions of Theorem 1, for any $f \in \mathrm{L}^{1}(\mathrm{~d} x \mathrm{~d} \mu) \cap \mathrm{L}^{2}(\mathrm{~d} x \mathrm{~d} v)$,

$$
\langle\text { АТ } \Pi f, \Pi f\rangle \geq \Phi\left(\|\Pi f\|^{2}\right)
$$

with

$$
\Phi^{-1}(y):=2 y+\left(\frac{y}{\mathrm{c}}\right)^{\frac{d}{d+2}}, \quad \mathrm{c}=\Theta \mathcal{C}_{\mathrm{Nash}}^{-\frac{d+2}{d}}\|f\|_{\mathrm{L}^{1}(\mathrm{~d} x \mathrm{~d} v)}^{-\frac{4}{d}}
$$

Proof. With $u$ defined by (13), we control $\|\Pi f\|^{2}=\left\|\rho_{f}\right\|_{\mathrm{L}^{2}(\mathrm{~d} x)}^{2}$ by $\langle$ AT $\Pi f, \Pi f\rangle$ according to

$$
\|\Pi f\|^{2}=\|u\|_{\mathrm{L}^{2}(\mathrm{~d} x)}^{2}+2 \Theta\|\nabla u\|_{\mathrm{L}^{2}(\mathrm{~d} x)}^{2}+\Theta^{2}\|\Delta u\|_{\mathrm{L}^{2}(\mathrm{~d} x)}^{2} \leq\|u\|_{\mathrm{L}^{2}(\mathrm{~d} x)}^{2}+2\langle\operatorname{AT\Pi } f, f\rangle
$$

using (14). Then we observe that

$$
\|u\|_{\mathrm{L}^{1}}=\left\|\rho_{f}\right\|_{\mathrm{L}^{1}}=\|f\|_{\mathrm{L}^{1}(\mathrm{~d} x \mathrm{~d} v)}, \quad\|u\|_{\mathrm{L}^{2}(\mathrm{~d} x)}^{2} \leq \frac{1}{\Theta}\langle\operatorname{AT} \Pi f, f\rangle
$$


and use Nash's inequality

$$
\|u\|_{\mathrm{L}^{2}(\mathrm{~d} x)}^{2} \leq \mathcal{C}_{\mathrm{Nash}}\|u\|_{\mathrm{L}^{1}(\mathrm{~d} x)}^{\frac{4}{d+2}}\|\nabla u\|_{\mathrm{L}^{2}(\mathrm{~d} x)}^{\frac{2 d}{d+2}}
$$

to conclude the proof.

The control of $(\mathrm{Id}-\Pi) f$ by the entropy production term relies on a simple new estimate.

Lemma 8. Under the assumptions of Theorem 1, for any solution $f$ of (1) with initial datum $f^{\text {in }} \in \mathrm{L}^{2}\left(\langle v\rangle^{k} \mathrm{~d} x \mathrm{~d} \mu\right) \cap \mathrm{L}^{1}(\mathrm{~d} x \mathrm{~d} v)$, we have

$$
\|(\mathrm{Id}-\Pi) f(t, \cdot, \cdot)\|_{-\beta}^{2} \geq \Psi\left(\|(\mathrm{Id}-\Pi) f(t, \cdot, \cdot)\|^{2}\right)
$$

for any $t \geq 0$, where $\mathcal{K}_{k}$ is as in Proposition 4 and

$$
\Psi(y):=C_{0} y^{1+\beta / k}, \quad C_{0}:=\left(\mathcal{K}_{k}\left(1+\Theta_{k}\right)\left\|f^{\text {in }}\right\|_{k}\right)^{-\frac{2 \beta}{k}} .
$$

Proof. Hölder's inequality

$$
\|(\mathrm{Id}-\Pi) f\| \leq\|(\mathrm{Id}-\Pi) f\|_{-\beta}^{\frac{k}{k+\beta}}\|(\mathrm{Id}-\Pi) f\|_{k}^{\frac{\beta}{k+\beta}}
$$

and

$$
\|(\mathrm{Id}-\text { П }) f\left\|_{k} \leq\right\| f\left\|_{k}+\Theta_{k}\right\| \rho\left\|_{\mathrm{L}^{2}(\mathrm{~d} x)} \leq\left(1+\Theta_{k}\right)\right\| f\left\|_{k} \leq \mathcal{K}_{k}\left(1+\Theta_{k}\right)\right\| f^{\text {in }} \|_{k},
$$

where the last inequality holds by Proposition 4, provide us with the estimate.

Proof of Theorem 1. Using the estimates of Lemma 7 and Lemma 8, we obtain that

$$
\|(\mathrm{Id}-\Pi) f\|_{-\beta}^{2}+\langle\operatorname{AT} \Pi f, \Pi f\rangle \geq \Psi\left(\|(\mathrm{Id}-\Pi) f\|^{2}\right)+\Phi\left(\|\Pi f\|^{2}\right) .
$$

Using (11) and the fact that $\mathrm{D}[f] \geq 0$ by Proposition 3 , we know that

$$
\|(\mathbf{I d}-\Pi) f\|^{2} \leq z_{0} \quad \text { and } \quad\|\Pi f\|^{2} \leq z_{0} \quad \text { where } \quad z_{0}:=\left\|f^{\text {in }}\right\|^{2} .
$$

Thus, from

$$
\Phi^{-1}(y)=2 y+\left(\frac{y}{c}\right)^{\frac{d}{d+2}} \leq\left(C_{1}^{-1} y\right)^{\frac{d}{d+2}} \quad \text { with } \quad C_{1}:=\left(2 \Phi\left(z_{0}\right)^{\frac{2}{d+2}}+\mathrm{c}^{-\frac{d}{d+2}}\right)^{-\frac{d+2}{d}},
$$

as long as $y \leq \Phi\left(z_{0}\right)$, we obtain

$$
\Phi\left(\|\Pi f\|^{2}\right) \geq C_{1}\|\Pi f\|^{2 \frac{d+2}{d}},
$$

since $\|\Pi f\|^{2} \leq z_{0}$. As a consequence,

$$
\begin{aligned}
&\|(\mathrm{Id}-\Pi) f\|_{-\beta}^{2}+\langle\operatorname{AT} \Pi f, \Pi f\rangle \geq C_{0}\|(\mathrm{Id}-\Pi) f\|^{2 \frac{k+\beta}{k}}+C_{1}\|\Pi f\|^{2 \frac{d+2}{d}} \\
& \geq \min \left\{C_{0} z_{0}^{\frac{\beta}{k}-\frac{1}{\zeta}}, C_{1} z_{0}^{\frac{2}{d}-\frac{1}{\zeta}}\right\}\|f\|^{2+\frac{2}{\zeta}}
\end{aligned}
$$


where $1 / \zeta=\max \{2 / d, \beta / k\}$, i.e., $\zeta=\min \{d / 2, k / \beta\}$. Collecting terms, we have

$$
\frac{\mathrm{d}}{\mathrm{d} t} \mathrm{H}[f] \leq-C \zeta \mathrm{H}[f]^{1+\frac{1}{\zeta}}
$$

using (10), (11) and Proposition 3, with

$$
C:=\frac{\kappa}{\zeta} \min \left\{C_{0} z_{0}^{\frac{\beta}{k}-\frac{1}{\zeta}}, C_{1} z_{0}^{\frac{2}{d}-\frac{1}{\zeta}}\right\}\left(\frac{2}{1+\delta}\right)^{1+\frac{1}{\zeta}}
$$

Then the result of Theorem 1 follows from a Grönwall estimate.

$$
\mathrm{H}[f(t, \cdot, \cdot)] \leq \mathrm{H}\left[f^{\text {in }}\right]\left(1+C \mathrm{H}\left[f^{\text {in }}\right]^{\frac{1}{\zeta}} t\right)^{-\zeta}
$$

The expression of $C$ can be explicitly computed in terms of $C_{0} z_{0}^{\frac{\beta}{k}-\frac{1}{\zeta}} \mathrm{H}\left[f^{\text {in }}\right]^{\frac{1}{\zeta}}$, which is proportional to $\left(\left\|f^{\text {in }}\right\| /\left\|f^{\text {in }}\right\|_{k}\right)^{\frac{2 \beta}{k}}$, and in terms of $C_{1} z_{0}^{\frac{2}{d}-\frac{1}{\zeta}} \mathrm{H}\left[f^{\text {in }}\right]^{\frac{1}{\zeta}}$ which is a function of $\left(\left\|f^{\text {in }}\right\|_{\mathrm{L}^{1}(\mathrm{~d} x \mathrm{~d} v)} /\left\|f^{\mathrm{in}}\right\|\right)^{4 /(d+2)}$, but it is of no practical interest. To see this, one has to take into account the expressions of $C_{0}, C_{1}$ and $\mathrm{c}$ in terms of the initial datum $f^{\text {in }}$.

As a concluding remark, we emphasize that a control of the solution in the space $\mathrm{L}^{2}\left(\langle v\rangle^{k} \mathrm{~d} x \mathrm{~d} \mu\right)$, based on Proposition 4, is enough to prove Theorem 1. In particular, there is no need of a uniform bound on $f$. This observation is new in $\mathrm{L}^{2}$ hypocoercive methods, and consistent with the homogeneous case (see Appendix B).

\section{Appendix A. Weighted Poincaré inequalities}

This appendix is devoted to a proof of (9) and considerations on related Poincaré inequalities. Inequality (9) is not a standard weighted Poincaré inequality because the average in the right-hand side of the inequality involves the measure of the left-hand side so that the right-hand side cannot be interpreted as a variance. Here we prove a generalization of (9) which relies on a purely spectral approach.

A.1. Continuous spectrum and weighted Poincaré inequalities. Let us consider two probability measures on $\mathbb{R}^{d}$

$$
\mathrm{d} \xi=e^{-\phi} \mathrm{d} v \quad \text { and } \quad \mathrm{d} \nu=\psi \mathrm{d} \xi
$$

where $\phi$ and $\psi \geq 0$ are two measurable functions, and the weighted Poincaré inequality

$$
\forall h \in \mathcal{D}\left(\mathbb{R}^{d}\right), \quad \int_{\mathbb{R}^{d}}|\nabla h|^{2} \mathrm{~d} \xi \geq \mathcal{C}_{\star} \int_{\mathbb{R}^{d}}|h-\widehat{h}|^{2} \mathrm{~d} \nu
$$

where $\widehat{h}=\int_{\mathbb{R}^{d}} h \mathrm{~d} \nu$. The question we address here is: on which conditions on $\phi$ and $\psi$ do we know that (20) holds for some constant $\mathcal{C}_{\star}>0$ ? Our key example is

$$
\phi(v)=\langle v\rangle^{\alpha}+\log Z_{\alpha} \quad \text { and } \quad \psi(v)=c_{\alpha, \beta}^{-1}\langle v\rangle^{-\beta}
$$

with $\alpha>0, \beta>0, Z_{\alpha}=\int_{\mathbb{R}^{d}} e^{-\phi} \mathrm{d} v$ and $c_{\alpha, \beta}=\int_{\mathbb{R}^{d}}\langle v\rangle^{-\beta} \mathrm{d} \xi$. 
Let us consider a potential $\Phi$ on $\mathbb{R}^{d}$ and assume that it is a measurable function with

$$
\sigma=\lim _{r \rightarrow+\infty} \inf _{w \in \mathcal{D}\left(B_{r}^{c}\right) \backslash\{0\}} \frac{\int_{\mathbb{R}^{d}}\left(|\nabla w|^{2}+\Phi|w|^{2}\right) \mathrm{d} v}{\int_{\mathbb{R}^{d}}|w|^{2} \mathrm{~d} v}>0,
$$

where $B_{r}^{c}:=\left\{v \in \mathbb{R}^{d}:|v|>r\right\}$ and $\mathcal{D}\left(B_{r}^{c}\right)$ denotes the space of smooth functions on $\mathbb{R}^{d}$ with compact support in $B_{r}^{c}$. According to Persson's result [21, Theorem 2.1], the lower end $\sigma_{\star}$ of the continuous spectrum of the Schrödinger operator $-\Delta+\Phi$ is such that

$$
\sigma_{\star} \geq \sigma \geq \lim _{r \rightarrow+\infty} \operatorname{infess}_{v \in B_{r}^{c}} \Phi(v) .
$$

If we replace $\int_{\mathbb{R}^{d}}|w|^{2} \mathrm{~d} v$ by the weighted integral $\int_{\mathbb{R}^{d}}|w|^{2} \psi \mathrm{d} v$ for some measurable function $\psi$, we have the modified result that the operator $\mathcal{L}=\psi^{-1}(-\Delta+\Phi)$ on $\mathrm{L}^{2}\left(\mathbb{R}^{d}, \psi \mathrm{d} v\right)$, associated with the quadratic form

$$
w \mapsto \int_{\mathbb{R}^{d}}\left(|\nabla w|^{2}+\Phi|w|^{2}\right) \mathrm{d} v
$$

has only discrete eigenvalues in the interval $(-\infty, \sigma)$ where

$$
\sigma=\lim _{r \rightarrow+\infty} \inf _{w \in \mathcal{D}\left(B_{r}^{c}\right) \backslash\{0\}} \frac{\int_{\mathbb{R}^{d}}\left(|\nabla w|^{2}+\Phi|w|^{2}\right) \mathrm{d} v}{\int_{\mathbb{R}^{d}}|w|^{2} \psi \mathrm{d} v}>0 .
$$

To prove it, it is enough to observe that 0 is the lower end of the continuous spectrum of $\mathcal{L}-\sigma_{\star}$ and to apply again [21, Theorem 2.1]. It is also straightforward to check that the lower end of the continuous spectrum of $\mathcal{L}$ is such that

$$
\sigma_{\star} \geq \lim _{r \rightarrow+\infty} \mathrm{q}(r)=: \sigma_{0} \quad \text { where } \quad \mathrm{q}(r):=\operatorname{infess}_{B_{r}^{c}} \frac{\Phi}{\psi} .
$$

Notice that $\sigma_{0}$ is either finite or infinite. In the case of $(21)$, we get that $\sigma_{0} \in(0,+\infty]$ if and only if $\beta \geq 2(1-\alpha)$. Relating the weighted Poincaré inequality (20) with the spectrum of $\mathcal{L}$ is then classical. Let

$$
h=w e^{\phi / 2}, \quad \Phi=\frac{1}{4}|\nabla \phi|^{2}-\frac{1}{2} \Delta \phi
$$

and observe that

$$
\begin{aligned}
& \int_{\mathbb{R}^{d}}|\nabla h|^{2} \mathrm{~d} \xi=Z_{\alpha}^{-1} \int_{\mathbb{R}^{d}}\left(|\nabla w|^{2}+\Phi|w|^{2}\right) \mathrm{d} v, \\
& \int_{\mathbb{R}^{d}}|h-\widehat{h}|^{2} \mathrm{~d} \xi=Z_{\alpha}^{-1} \int_{\mathbb{R}^{d}}|w-\widetilde{w}|^{2} \psi \mathrm{d} v,
\end{aligned}
$$

where $\widetilde{w}=\frac{\int_{\mathbb{R}^{d}} w \psi e^{-\phi / 2} \mathrm{~d} v}{\int_{\mathbb{R}^{d}} \psi e^{-\phi} \mathrm{d} v} e^{-\phi / 2}$.

Proposition 9. With the above notations, let $\Phi$ and $\psi$ be two measurable functions such that $\sigma_{0}>0$. Then inequality (20) holds for some positive, finite, optimal constant $\mathcal{C}_{\star}>0$. Otherwise, if we have that $\lim _{r \rightarrow+\infty} \operatorname{supess}_{v \in B_{r}^{c}} \frac{\Phi(v)}{\psi(v)}=0$, then inequality (20) does not hold. 
Proof. By construction, $\sigma$ is nonnegative and the infimum of the Rayleigh quotient

$$
w \mapsto \frac{\int_{\mathbb{R}^{d}}\left(|\nabla w|^{2}+\Phi|w|^{2}\right) \mathrm{d} v}{\int_{\mathbb{R}^{d}}|w|^{2} \psi \mathrm{d} v}
$$

is achieved by $h \equiv \widehat{h}=1$, that is, by $w=\widetilde{w}=e^{-\phi / 2}$, which moreover generates the kernel of $\mathcal{L}$. Hence we can interpret $\mathcal{C}_{\star}$ as the first positive eigenvalue, if there is any in the interval $\left(0, \sigma_{\star}\right)$, or $\mathcal{C}_{\star}=\sigma_{\star}$ if there is none.

In the case of $(21)$, the condition $\beta \geq 2(1-\alpha)$ is a necessary and sufficient condition for the inequality (20) to hold. The threshold case $\beta=2(1-\alpha)$ is remarkable: inequality $(20)$ can be rewritten for any $\alpha \in(0,1)$ as the following weighted Poincaré inequality :

$$
\forall h \in \mathcal{D}\left(\mathbb{R}^{d}\right), \quad \int_{\mathbb{R}^{d}}|\nabla h|^{2} e^{-\langle v\rangle^{\alpha}} \mathrm{d} v \geq \mathcal{C}_{\star} \int_{\mathbb{R}^{d}} \frac{|h-\widehat{h}|^{2} e^{-\langle v\rangle^{\alpha}}}{\left(1+|v|^{2}\right)^{1-\alpha}} \mathrm{d} v,
$$

for some constant $\mathcal{C}_{\star} \in\left(0, \alpha^{2} / 4\right]$ and

$$
\widehat{h}:=\frac{1}{z_{\alpha}} \int_{\mathbb{R}^{d}} \frac{h e^{-\langle v\rangle^{\alpha}}}{\left(1+|v|^{2}\right)^{1-\alpha}} \mathrm{d} v, \quad z_{\alpha}=\int_{\mathbb{R}^{d}} \frac{e^{-\langle v\rangle^{\alpha}}}{\left(1+|v|^{2}\right)^{1-\alpha}} \mathrm{d} v .
$$

\section{A.2. A weighted Poincaré inequality with a non-classical average.}

Corollary 10. Let $\Phi$ and $\psi$ be respectively a measurable function and a bounded positive function such that, with the notations of section $A .1, \sigma_{0}>0$ and $\psi^{-1} \in$ $\mathrm{L}^{1}\left(\mathbb{R}^{d}, \mathrm{~d} \xi\right)$. Then the inequality

$$
\forall h \in \mathcal{D}\left(\mathbb{R}^{d}\right), \quad \int_{\mathbb{R}^{d}}|\nabla h|^{2} \mathrm{~d} \xi \geq \mathcal{C} \int_{\mathbb{R}^{d}}|h-\widetilde{h}|^{2} \mathrm{~d} \nu
$$

holds for some optimal constant $\mathcal{C} \in\left(0, \mathcal{C}_{\star}\right]$, where $\widetilde{h}:=\int_{\mathbb{R}^{d}} h \mathrm{~d} \xi$. Here $\mathcal{C}_{\star}$ denotes the optimal constant in (20).

As we shall see in the proof, our method provides us with an explicit lower bound $\mathcal{C}$ in terms of $\mathcal{C}_{\star}$. We emphasize that in (24), the right-hand side is not the variance of $h$ with respect of the measure $\mathrm{d} \nu$ because we subtract the average with respect to the measure $\mathrm{d} \xi$ which appears in the left-hand side. In case $\phi(v)=\langle v\rangle^{\alpha}$, inequality (24) is equivalent to [14, inequality (1.12)], which can be deduced using the strategy of $[1,2]$. Also see Appendix B.1 for further details.

Proof. Let us consider a function $h$. With no loss of generality, we can assume that $\widetilde{h}=\int_{\mathbb{R}^{d}} h \mathrm{~d} \xi=0$ up to the replacement of $h$ by $h-\widetilde{h}$. We use the IMS decomposition method (see [20, 23]), which goes as follows. Let $\chi$ be a truncation function on $\mathbb{R}_{+}$ with the following properties: $0 \leq \chi \leq 1, \chi \equiv 1$ on $[0,1], \chi \equiv 0$ on $[2,+\infty)$ and $\chi^{\prime 2} /\left(1-\chi^{2}\right) \leq \kappa$ for some $\kappa>0$. Next, we define $\chi_{R}(v)=\chi(|v| / R), h_{1, R}=h \chi_{R}$ 
and $h_{2, R}=h \sqrt{1-\chi_{R}^{2}}$, so that $h_{1, R}$ is supported in the ball $B_{2 R}$ of radius $2 R$ centered at $v=0$ and $h_{2, R}$ is supported in $B_{R}^{c}=\mathbb{R}^{d} \backslash B_{R}$. Elementary computations show that $h^{2}=h_{1, R}^{2}+h_{2, R}^{2}$ and $|\nabla h|^{2}=\left|\nabla h_{1, R}\right|^{2}+\left|\nabla h_{2, R}\right|^{2}-h^{2}|\nabla \chi|^{2} /\left(1-\chi^{2}\right)$, so that $\left.|| \nabla h\right|^{2}-\left|\nabla h_{1, R}\right|^{2}-\left|\nabla h_{2, R}\right|^{2} \mid \leq \kappa h^{2} / R^{2}$.

Since $h_{2, R}$ is supported in $B_{R}^{c}$, we know that

$$
\int_{\mathbb{R}^{d}}\left|\nabla h_{2, R}\right|^{2} \mathrm{~d} \xi \geq \mathrm{q}(R) \int_{\mathbb{R}^{d}}\left|h_{2, R}\right|^{2} \mathrm{~d} \nu
$$

for any $R>0$, where $\mathrm{q}$ is the quotient involved in the definition of $\sigma_{0}$. We recall that $\lim _{r \rightarrow+\infty} \mathbf{q}(r)=\sigma_{0}>0$. Using the method of the Holley-Stroock lemma (see [12] and [8] for a recent presentation), we deduce from inequality (20) that

$$
\begin{aligned}
\int_{\mathbb{R}^{d}}\left|\nabla h_{1, R}\right|^{2} \mathrm{~d} \xi & \geq \mathcal{C}_{\star} \int_{\mathbb{R}^{d}}\left|h_{1, R}-\widehat{h}_{1, R}\right|^{2} \mathrm{~d} \nu \\
& \geq \mathcal{C}_{\star} \int_{B_{2 R}}\left|h_{1, R}-\widehat{h}_{1, R}\right|^{2} \psi \mathrm{d} \xi \\
& \geq \mathcal{C}_{\star} \inf _{B_{2 R}} \psi \min _{c \in \mathbb{R}} \int_{B_{2 R}}\left|h_{1, R}-c\right|^{2} \mathrm{~d} \xi \\
& \geq \mathrm{Q}(R) \int_{\mathbb{R}^{d}}\left|h_{1, R}\right|^{2} \mathrm{~d} \nu-\mathcal{C}_{\star} \frac{\inf _{B_{2 R}} \psi}{\xi\left(B_{2 R}\right)}\left(\int_{\mathbb{R}^{d}} h_{1, R} \mathrm{~d} \xi\right)^{2}
\end{aligned}
$$

where $\mathrm{Q}(R):=\mathcal{C}_{\star} \inf _{B_{2 R}} \psi / \sup _{B_{2 R}} \psi$. By the assumption $\widetilde{h}=0$, we know that

$$
\int_{B_{R}} h \mathrm{~d} \xi=-\int_{B_{R}^{c}} h \mathrm{~d} \xi
$$

from which we deduce that

$$
\left(\int_{\mathbb{R}^{d}} h_{1, R} \mathrm{~d} \xi\right)^{2}=\left(\int_{B_{R}} h \mathrm{~d} \xi+\int_{B_{R}^{c}} \chi h \mathrm{~d} \xi\right)^{2} \leq\left(\int_{B_{R}^{c}}|h| \mathrm{d} \xi\right)^{2} \leq \int_{\mathbb{R}^{d}} h^{2} \mathrm{~d} \nu \int_{B_{R}^{c}} \psi^{-1} \mathrm{~d} \xi
$$

where the last inequality is simply a Cauchy-Schwarz inequality. Let

$$
\varepsilon(R):=\mathcal{C}_{\star} \frac{\inf _{B_{2 R}} \psi}{\xi\left(B_{2 R}\right)} \int_{B_{R}^{c}} \psi^{-1} \mathrm{~d} \xi .
$$

By the assumption that $\psi^{-1} \in \mathrm{L}^{1}\left(\mathbb{R}^{d}, \mathrm{~d} \xi\right)$, we know that

$$
\lim _{R \rightarrow+\infty} \varepsilon(R)=0 \quad \text { and } \quad \lim _{R \rightarrow+\infty} \frac{\varepsilon(R)}{\mathrm{Q}(R)}=0 .
$$

Collecting all our assumptions, we have

$$
\begin{aligned}
\int_{\mathbb{R}^{d}}|\nabla h|^{2} \mathrm{~d} \xi & \geq \int_{\mathbb{R}^{d}}\left(\left|\nabla h_{1, R}\right|^{2}+\left|\nabla h_{2, R}\right|^{2}-\frac{\kappa}{R^{2}} h^{2}\right) \mathrm{d} \xi \\
& \geq\left(\min \{\mathrm{Q}(R), \mathrm{q}(R)\}-\varepsilon(R)-\frac{\kappa}{R^{2}}\right) \int_{\mathbb{R}^{d}}|h|^{2} \mathrm{~d} \nu
\end{aligned}
$$

where $\min \{\mathrm{Q}(R), \mathrm{q}(R)\}-\varepsilon(R)-\kappa / R^{2}$ is positive for $R>0$, large enough. 
Finally, let us notice that for any $c \in \mathbb{R}$ we have

$$
\int_{\mathbb{R}^{d}}|h-c|^{2} \mathrm{~d} \nu=\int_{\mathbb{R}^{d}} h^{2} \mathrm{~d} \nu-2 c \int_{\mathbb{R}^{d}} h \mathrm{~d} \nu+c^{2} \geq \int_{\mathbb{R}^{d}}|h-\widehat{h}|^{2} \mathrm{~d} \nu
$$

with equality if and only if $c=\widehat{h}=\int_{\mathbb{R}^{d}} h \mathrm{~d} \nu$. As a special case corresponding to $c=\widetilde{h}=\int_{\mathbb{R}^{d}} h \mathrm{~d} \xi$, we have

$$
\int_{\mathbb{R}^{d}}|h-\widetilde{h}|^{2} \mathrm{~d} \nu \geq \int_{\mathbb{R}^{d}}|h-\widehat{h}|^{2} \mathrm{~d} \nu
$$

This proves that $\mathcal{C}_{\star} \geq \mathcal{C}$.

In the special case of $(21)$, it is possible to give a slightly shorter proof using the Poincaré inequality on $B_{R}$, for the measure $\mathrm{d} \xi$ : see [18, Chapter 6]. An independent proof of such an inequality is anyway needed for a general $\phi$. The proof of Corollary 10 is more general and reduces everything to a comparison of the asymptotic behavior of $\phi$ and $\psi$. If these functions are given by (21), inequality (24) can be rewritten in the form of (9), we have an estimate of $\mathcal{C}$ and we can characterize $\mathcal{C}_{\star}$ as follows.

Proposition 11. The optimal constant $\mathcal{C}_{\star}$ is the ground state energy of the operator $\mathcal{L}=\psi^{-1}(-\Delta+\Phi)$ on $\mathrm{L}^{2}\left(\mathbb{R}^{d}, \psi \mathrm{d} v\right)$.

The proof relies on (22). Details are left to the reader.

\section{Appendix B. Algebraic decay rates for the Fokker-Planck equation}

Here we consider simple estimates of the decay rates in the homogeneous case given by $f(t, x, v)=g(t, v)$ of equation (1), that is, the Fokker-Planck equation

$$
\partial_{t} g=\mathrm{L}_{1} g \text {. }
$$

After summarizing the standard approach based on the weak Poincaré inequality (see for instance [14]) in Section B.1, we introduce a new method which relies on weighted $\mathrm{L}^{2}$ estimates. As already mentioned, the advantage of weighted Poincaré inequalities is that the description of the convergence rates to the local equilibrium does not require extra regularity assumptions to cover the transition from super-exponential $(\alpha>1)$ and exponential $(\alpha=1)$ local equilibria to sub-exponential local equilibria, with $\alpha \in(0,1)$.

B.1. Weak Poincaré inequality. We assume $\alpha \in(0,1)$ and $\eta \in(0, \beta)$ with $\beta=$ $2(1-\alpha)$. By a simple Hölder inequality, with $(\tau+1) / \tau=\beta / \eta$, we obtain that

$$
\begin{aligned}
\int_{\mathbb{R}^{d}}|h-\widetilde{h}|^{2} \mathrm{~d} \xi= & \int_{\mathbb{R}^{d}}|h-\widetilde{h}|^{2}\langle v\rangle^{-\eta}\langle v\rangle^{\eta} \mathrm{d} \xi \\
& \leq\left(\int_{\mathbb{R}^{d}}|h-\widetilde{h}|^{2}\langle v\rangle^{-\beta} \mathrm{d} \xi\right)^{\frac{\tau}{\tau+1}}\left(\int_{\mathbb{R}^{d}}\|h-\widetilde{h}\|_{\mathrm{L}^{\infty}\left(\mathbb{R}^{d}\right)}^{2}\langle v\rangle^{\beta \tau} \mathrm{d} \xi\right)^{\frac{1}{1+\tau}} .
\end{aligned}
$$


Here we choose $\widetilde{h}:=\int_{\mathbb{R}^{d}} h \mathrm{~d} \xi$. Using (9), we end up with

$$
\forall h \in \mathcal{D}\left(\mathbb{R}^{d}\right), \quad \int_{\mathbb{R}^{d}}|h-\widetilde{h}|^{2} \mathrm{~d} \xi \leq \mathcal{C}_{\alpha, \tau}\left(\int_{\mathbb{R}^{d}}|\nabla h|^{2} \mathrm{~d} \xi\right)^{\frac{\tau}{1+\tau}}\|h-\widetilde{h}\|_{\mathrm{L}^{\infty}\left(\mathbb{R}^{d}\right)}^{\frac{2}{1+\tau}},
$$

for some explicit positive constant $\mathcal{C}_{\alpha, \tau}$. We learn from (5) that

$$
\frac{\mathrm{d}}{\mathrm{d} t} \int_{\mathbb{R}^{d}}|h(t, \cdot)-\widetilde{h}|^{2} \mathrm{~d} \xi=-2 \int_{\mathbb{R}^{d}}\left|\nabla_{v} h\right|^{2} \mathrm{~d} \xi
$$

if $g=h F$ is solves (25), and we also know that $\widetilde{h}$ does not depend on $t$. By a strategy that goes back at least to [16, Theorem 2.2] and is due, according to the author, to D. Stroock, we obtain that

$$
\int_{\mathbb{R}^{d}}|h(t, \cdot)-\widetilde{h}|^{2} \mathrm{~d} \xi \leq\left(\left(\int_{\mathbb{R}^{d}}|h(0, \cdot)-\widetilde{h}|^{2} \mathrm{~d} \xi\right)^{-\frac{1}{\tau}}+\frac{2 \tau^{-1}}{\mathcal{C}_{\alpha, \tau}^{1+1 / \tau} \mathcal{M}} t\right)^{-\tau}
$$

with $\mathcal{M}=\sup _{s \in(0, t)}\|h(s, \cdot)-\widetilde{h}\|_{L^{\infty}\left(\mathbb{R}^{d}\right)}^{2 / \tau}$. The limitation is of course that we need to restrict the initial conditions in order to have $\mathcal{M}$ uniformly bounded with respect to $t$. Since $\eta$ can be chosen arbitrarily close to $\beta$, the exponent $\tau$ can be taken arbitrarily large but to the price of a constant $\mathcal{C}_{\alpha, \tau}$ which explodes as $\eta \rightarrow \beta_{-}$.

Notice that (26) is equivalent to the weak Poincaré inequality

$$
\forall h \in \mathcal{D}\left(\mathbb{R}^{d}\right), \quad \mathcal{C}_{\alpha, \tau}^{-1} \int_{\mathbb{R}^{d}}|h-\widetilde{h}|^{2} \mathrm{~d} \xi \leq \frac{\tau}{(1+\tau)^{1+\frac{1}{\tau}}} r^{-\frac{\tau}{1+\tau}} \int_{\mathbb{R}^{d}}|\nabla h|^{2} \mathrm{~d} \xi+r\|h-\widetilde{h}\|_{\mathrm{L}^{\infty}\left(\mathbb{R}^{d}\right)}^{2},
$$

for all $r>0$, as stated in [22, (1.6) and example 1.4 (c)]. The equivalence of this inequality and (26) is easily recovered by optimizing on $r>0$. It is worth to remark that here we consider $\|h-\widetilde{h}\|_{L^{\infty}\left(\mathbb{R}^{d}\right)}$ while various other quantities like, e.g., the median can be used in weak Poincaré inequalities.

B.2. Weighted $\mathrm{L}^{2}$ estimates. As an alternative approach to the weak Poincaré inequality method of Appendix B.1, we can consider for some arbitrary $k>0$ the evolution according to equation (25) of $\int_{\mathbb{R}^{d}}|h(t, v)|^{2}\langle v\rangle^{k} \mathrm{~d} \xi=\int_{\mathbb{R}^{d}}|h(t, v)|^{2}\langle v\rangle^{k} F \mathrm{~d} v$ where $h:=g / F$ solves

$$
\partial_{t} h=F^{-1} \nabla_{v} \cdot\left(F \nabla_{v} h\right)
$$

Let us compute

$$
\frac{\mathrm{d}}{\mathrm{d} t} \int_{\mathbb{R}^{d}}|h(t, v)|^{2}\langle v\rangle^{k} F \mathrm{~d} v+2 \int_{\mathbb{R}^{d}}\left|\nabla_{v} h\right|^{2}\langle v\rangle^{k} F \mathrm{~d} v=-\int_{\mathbb{R}^{d}} \nabla_{v}\left(h^{2}\right) \cdot\left(\nabla_{v}\langle v\rangle^{k}\right) F \mathrm{~d} v
$$

and observe with $\ell=2-\alpha$ that

$$
\nabla_{v} \cdot\left(F \nabla_{v}\langle v\rangle^{k}\right)=\frac{k}{\langle v\rangle^{4}}\left(d+(k+d-2)|v|^{2}-\alpha\langle v\rangle^{\alpha}|v|^{2}\right) \leq a-b\langle v\rangle^{-\ell}
$$


for some $a \in \mathbb{R}, b \in(0,+\infty)$. The same proof as in Proposition 4 shows that there exists a constant $\mathcal{K}_{k}>0$ such that

$$
\forall t \geq 0 \quad\|h(t, \cdot)\|_{\mathrm{L}^{2}\left(\langle v\rangle^{k} \mathrm{~d} \xi\right)} \leq \mathcal{K}_{k}\left\|h^{\mathrm{in}}\right\|_{\mathrm{L}^{2}\left(\langle v\rangle^{k} \mathrm{~d} \xi\right)} .
$$

Hence, if $h$ solves (25) with initial value $h^{\text {in }}$, we can use (9) to write

$$
\frac{\mathrm{d}}{\mathrm{d} t} \int_{\mathbb{R}^{d}}|h(t, \cdot)-\widetilde{h}|^{2} \mathrm{~d} \xi=-2 \int_{\mathbb{R}^{d}}\left|\nabla_{v} h\right|^{2} \mathrm{~d} \xi \leq-2 \mathcal{C} \int_{\mathbb{R}^{d}}|h-\widetilde{h}|^{2}\langle v\rangle^{-\beta} \mathrm{d} \xi
$$

with $\beta=2(1-\alpha)$ and $\widetilde{h}=\int_{\mathbb{R}^{d}} h \mathrm{~d} \xi$. With $\theta=k /(k+\beta)$, Hölder's inequality

$$
\int_{\mathbb{R}^{d}}|h-\widetilde{h}|^{2} \mathrm{~d} \xi \leq\left(\int_{\mathbb{R}^{d}}|h-\widetilde{h}|^{2}\langle v\rangle^{-\beta} \mathrm{d} \xi\right)^{\theta}\left(\int_{\mathbb{R}^{d}}|h-\widetilde{h}|^{2}\langle v\rangle^{k} \mathrm{~d} \xi\right)^{1-\theta}
$$

allows us to estimate the right hand side and obtain the following result.

Proposition 12. Let $\alpha \in(0,1)$, let $g^{\text {in }} \in \mathrm{L}_{+}^{1}(\mathrm{~d} \mu) \cap \mathrm{L}^{2}\left(\langle v\rangle^{k} \mathrm{~d} \mu\right)$ for some $k>0$, and consider the solution $g$ to (25) with initial datum $g^{\text {in }}$. With $\mathcal{C}$ as in (9), if $\bar{g}=$ $\left(\int_{\mathbb{R}^{d}} g \mathrm{~d} v\right) F$ where $F$ is given by $(2)$, then

$$
\int_{\mathbb{R}^{d}}|g(t, \cdot)-\bar{g}|^{2} \mathrm{~d} \mu \leq\left(\left(\int_{\mathbb{R}^{d}}\left|g^{\mathrm{in}}-\bar{g}\right|^{2} \mathrm{~d} \mu\right)^{-\beta / k}+\frac{2 \beta \mathcal{C}}{k \mathcal{K}^{\beta / k}} t\right)^{-k / \beta}
$$

with $\beta=2(1-\alpha)$ and $\mathcal{K}:=\mathcal{K}_{k}^{2}\left\|g^{\mathrm{in}}\right\|_{\mathrm{L}^{2}\left(\langle v\rangle^{k} \mathrm{~d} \mu\right)}^{2}+\Theta_{k}\left(\int_{\mathbb{R}^{d}} g^{\mathrm{in}} \mathrm{d} v\right)^{2}$.

We recall that $g=h F, \bar{g}=\widetilde{h} F$ and $F \mathrm{~d} \mu=\mathrm{d} v=F^{-1} \mathrm{~d} \xi$. We notice that arbitrarily large decay rates can be obtained under the condition that $k>0$ is large enough. We recover that when $k<d \beta / 2$, the rate of relaxation to the equilibrium is slower than $(1+t)^{-d / 2}$ and responsible for the limitation that appears in Theorem 1. However, the rate of the heat flow is recovered in Theorem 1 for a weight of order $k$ with an arbitrarily small but fixed $k>0$, if $\alpha$ is taken close enough to 1 .

Proof. Using

$$
\frac{1}{2} \int_{\mathbb{R}^{d}}|h-\widetilde{h}|^{2}\langle v\rangle^{k} \mathrm{~d} \xi \leq \int_{\mathbb{R}^{d}}|h|^{2}\langle v\rangle^{k} \mathrm{~d} \xi+\Theta_{k} \widetilde{h}^{2}=\mathcal{K},
$$

we obtain that $y(t):=\int_{\mathbb{R}^{d}}|g(t, \cdot)-\bar{g}|^{2} \mathrm{~d} \mu$ obeys to $y^{\prime} \leq-2 \mathcal{C} \mathcal{K}^{1-1 / \theta} y^{1 / \theta}$ and conclude by a Grönwall estimate. 


\section{ACKNOWLEDGMENTS}

This work has been partially supported by the Project EFI (E.B., J.D., ANR-17-CE400030) of the French National Research Agency (ANR). Support by the Austrian Science Foundation (grants no. F65 and W1245) is acknowledged by C.S. Moreover C.S. is very grateful for the hospitality at Université Paris-Dauphine. E.B., J.D., L.L. and C.S. are participants to the Amadeus project Hypocoercivity no. 39453PH. All authors thank Clément Mouhot for stimulating discussions and encouragements.

(c) 2019 by the authors. This paper may be reproduced, in its entirety, for non-commercial purposes.

\section{REFERENCES}

[1] Bakry, D., Barthe, F., Cattiaux, P., and Gulllin, A. A simple proof of the Poincaré inequality for a large class of probability measures including the log-concave case. Electron. Commun. Probab. 13 (2008), 60-66.

[2] Bakry, D., Cattiaux, P., and Gulllin, A. Rate of convergence for ergodic continuous Markov processes: Lyapunov versus Poincaré. J. Funct. Anal. 254, 3 (2008), 727-759.

[3] Bakry, D., Gentil, I., And Ledoux, M. Analysis and geometry of Markov diffusion operators, vol. 348 of Grundlehren der Mathematischen Wissenschaften [Fundamental Principles of Mathematical Sciences]. Springer, Cham, 2014.

[4] Bouin, E., Dolbeault, J., Mischler, S., Mouhot, C., and Schmeiser, C. Hypocoercivity without confinement. Preprint hal-01575501 and arxiv: 170806180B, to appear in Pure and Applied Analysis, Oct. 2017.

[5] Bouin, E., Dolbeault, J., And Schmeiser, C. Diffusion and kinetic transport with very weak confinement. Preprint hal-01991665 and arxiv: 1901.08323, to appear in Kinetic Rel. Models, Jan. 2019.

[6] CAO, C. The kinetic Fokker-Planck equation with weak confinement force. Preprint hal01697058 and arxiv: 1801.10354, June 2018.

[7] Degond, P., Goudon, T., And Poupaud, F. Diffusion limit for nonhomogeneous and nonmicro-reversible processes. Indiana Univ. Math. J. 49, 3 (2000), 1175-1198.

[8] Dolbeault, J., And Li, X. $\Phi$-Entropies: convexity, coercivity and hypocoercivity for FokkerPlanck and kinetic Fokker-Planck equations. Mathematical Models and Methods in Applied Sciences 28, 13 (2018), 2637-2666.

[9] Dolbeault, J., Mouhot, C., And Schmeiser, C. Hypocoercivity for kinetic equations with linear relaxation terms. C. R. Math. Acad. Sci. Paris 347, 9-10 (2009), 511-516.

[10] Dolbeault, J., Mouhot, C., And Schmeiser, C. Hypocoercivity for linear kinetic equations conserving mass. Trans. Amer. Math. Soc. 367, 6 (2015), 3807-3828.

[11] Gualdani, M. P., Mischler, S., and Mouhot, C. Factorization of non-symmetric operators and exponential H-theorem. Mém. Soc. Math. Fr. (N.S.), 153 (2017), 137.

[12] Holley, R., And Stroock, D. Logarithmic Sobolev inequalities and stochastic Ising models. Journal of Statistical Physics 46, 5 (1987), 1159-1194.

[13] Hu, S., AND WANG, X. Subexponential decay in kinetic Fokker-Planck equation: weak hypocoercivity. Bernoulli 25, 1 (2019), 174-188.

[14] Kavian, O., AND MischleR, S. The Fokker-Planck equation with subcritical confinement force. Preprint hal-01241680 and arxiv: 1512.07005, Dec. 2016.

[15] Li, T., AND Zhang, Z. Large time behaviour for the Fokker-Planck equation with general potential. Sci. China Math. 61, 1 (2018), 137-150. 
[16] Liggett, T. M. $L_{2}$ rates of convergence for attractive reversible nearest particle systems: the critical case. Ann. Probab. 19, 3 (1991), 935-959.

[17] Mellet, A., Mischler, S., And Mouhot, C. Fractional diffusion limit for collisional kinetic equations. Arch. Ration. Mech. Anal. 199, 2 (2011), 493-525.

[18] MischleR, S. Book in preparation. Personnal communication.

[19] Mischler, S., And Mounot, C. Exponential stability of slowly decaying solutions to the kinetic-Fokker-Planck equation. Arch. Ration. Mech. Anal. 221, 2 (2016), 677-723.

[20] Morgan, III, J. D. Schrödinger operators whose potentials have separated singularities. J. Operator Theory 1, 1 (1979), 109-115.

[21] Persson, A. Bounds for the discrete part of the spectrum of a semi-bounded Schrödinger operator. Math. Scand. 8 (1960), 143-153.

[22] Röckner, M., AND WANG, F.-Y. Weak Poincaré inequalities and $\mathrm{L}^{2}$-convergence rates of Markov semigroups. J. Funct. Anal. 185, 2 (2001), 564-603.

[23] Simon, B. Semiclassical analysis of low lying eigenvalues. I. Nondegenerate minima: asymptotic expansions. Ann. Inst. H. Poincaré Sect. A (N.S.) 38, 3 (1983), 295-308.

[24] WANG, X. P. Large-time asymptotics of solutions to the Kramers-Fokker-Planck equation with a short-range potential. Communications in Mathematical Physics 336, 3 (Jan 2015), 1435-1471.

(Emeric Bouin) CEREMADE (CNRS UMR No 7534), PSL UNIVERSiTy, Université PARISDauphine, Place de Lattre de Tassigny, 75775 Paris 16, France

E-mail address: bouin@ceremade.dauphine.fr

(Jean Dolbeault) CEREMADE (CNRS UMR N 7534), PSL UNIVERSITY, Université PARISDauphine, Place de Lattre de Tassigny, 75775 Paris 16, France

E-mail address: dolbeaul@ceremade.dauphine.fr

(Laurent Lafleche) CEREMADE (CNRS UMR N 7534), PSL UNIVERSiTy, Université PARISDauphine, Place de Lattre de Tassigny, 75775 Paris 16, France

E-mail address: lafleche@ceremade.dauphine.fr

(Christian Schmeiser) Fakultät für Mathematik, Universität Wien, Oskar-MorgensternPlatz 1, 1090 Wien, Austria

E-mail address: Christian.Schmeiser@univie.ac.at 